\title{
Monosynaptic Pathway from Rat Vibrissa Motor Cortex to Facial Motor Neurons Revealed by Lentivirus-Based Axonal Tracing
}

\author{
Valery Grinevich, ${ }^{1}$ Michael Brecht, ${ }^{2}$ and Pavel Osten ${ }^{1}$ \\ Departments of ${ }^{1}$ Molecular Neurobiology and ${ }^{2}$ Cell Physiology, Max Planck Institute for Medical Research, 69120 Heidelberg, Germany
}

The mammalian motor cortex typically innervates motor neurons indirectly via oligosynaptic pathways. However, evolution of skilled digit movements in humans, apes, and some monkey species is associated with the emergence of abundant monosynaptic cortical projections onto spinal motor neurons innervating distal limb muscles. Rats perform skilled movements with their whiskers, and we examined the possibility that the rat vibrissa motor cortex (VMC) projects monosynaptically onto facial motor neurons controlling the whisker movements. First, single injections of lentiviruses to VMC sites identified by intracortical microstimulations were used to label a distinct subpopulation of VMC axons or presynaptic terminals by expression of enhanced green fluorescent protein (GFP) or GFPtagged synaptophysin, respectively. Four weeks after the injections, GFP and synaptophysin-GFP labeling of axons and putative presynaptic terminals was detected in the lateral portion of the facial nucleus (FN), in close proximity to motor neurons identified morphologically and by axonal back-labeling from the whisker follicles. The VMC projections were detected bilaterally, with threefold larger density of labeling in the contralateral FN. Next, multiple VMC injections were used to label a large portion of VMC axons, resulting in overall denser but still laterally restricted FN labeling. Ultrastructural analysis of the GFP-labeled VMC axons confirmed the existence of synaptic contacts onto dendrites and somata of FN motor neurons. These findings provide anatomical demonstration of monosynaptic VMCto-FN pathway in the rat and show that lentivirus-based expression of GFP and GFP-tagged presynaptic proteins can be used as a high-resolution neuroanatomical tracing method.

Key words: vibrissa motor cortex; motor neuron; facial nucleus; lentivirus; GFP; axonal tracing

\section{Introduction}

Active positioning of sensors is characteristic of virtually all sensory systems, and such exploratory movements are thought to play a crucial role in the vibrissal system of rodents (Welker, 1964; Lee and Woolsey, 1975; Wineski, 1985; Carvell and Simons, 1990; Brecht et al., 1997). Some rodents (e.g., rats, hamsters, and mice) generate rhythmic high-speed whisker movements called "whisking." Although such movements are typically synchronous, small divergent movements of adjacent whiskers have been described in rats (Sachdev et al., 2002). Whisker protractions are mediated by intrinsic whisker muscles forming a sling around the base of each whisker (Dörfl, 1982), whereas whisker retractions are driven by extrinsic whisker muscles running underneath the

Received Jan. 18, 2005; revised July 25, 2005; accepted July 25, 2005.

This work was supported by the Max Planck Society and by VIDI and Human Frontier Science Program grants to M.B. We thank Peter H. Seeburg for generous support. We thank Marina Eliava for help with electron microscopy; Hilmar Bading for kindly allowing us to use the electron microscopy facility; Guenter Giesse for help with confocal microscopy; and Sabine Gruenewald, Carmen Grosskurth, and Judith Müller for help with lentivirus production. The synaptophysin-GFP construct was a kind gift from Rudolf Leube. We thank Bert Sakmann, Lucas Herfst, and Albert Lee for comments on this manuscript.

Correspondence should be addressed to Pavel 0sten, Department of Molecular Neurobiology, Max Planck Institute for Medical Research, Jahnstrasse29,69120 Heidelberg, Germany. E-mail: posten@mpimf-heidelberg.mpg.de.

M. Brecht's present address: Department of Neuroscience, Erasmus Medical Center, Postbus 1738, 3000 DR Rotterdam, The Netherlands.

DOI:10.1523/JNEUROSCI.2235-05.2005

Copyright $\odot 2005$ Society for Neuroscience $\quad 0270-6474 / 05 / 258250-09 \$ 15.00 / 0$ skin (Wineski, 1985; Berg and Kleinfeld, 2003). These muscles are highly specialized in the whisking rodents, with a predominance of fast-contracting, fast-fatigable, type $2 \mathrm{~B}$ muscle fibers (Jin et al., 2004).

Given the great behavioral significance of whiskers, it is not surprising that a large cortical motor field, the vibrissa motor cortex (VMC), is devoted to controlling these movements (Fig. 1A) (Hall and Lindholm, 1974). The VMC area takes up $\sim 45 \%$ of the entire rat primary motor cortex (M1) and $\sim 4.5 \%$ of the total volume of the rat neocortex and allocortex (Wree et al., 1992; Brecht et al., 2004b). Notably, extracellular microstimulation of the VMC can evoke movements restricted to one or two whiskers (Izraeli and Porter, 1995; Brecht et al., 2004b), indicating a functional link between a focal VMC activation and movements of individual whiskers. However, somewhat counter-intuitively to this evidence, two previous anatomical studies described only indirect connections from the VMC to facial motor neurons (Miyashita et al., 1994; Hattox et al., 2002).

Anatomical, developmental, and comparative studies in humans and monkeys indicate that direct motor cortex projections onto spinal cord motor neurons have evolved to allow a high degree of control over contractions of orofacial, tongue, and finger muscles (Kuypers, 1982; Porter and Lemon, 1995). Intrigued by the findings that rodents can actively position their individual whiskers (Sachdev et al., 2002, 2003) and that individual whisker 
movements can be evoked by intracortical microstimulation (Izraeli and Porter, 1995; Brecht et al., 2004b), we reexamined the possibility that the VMC projects directly to facial nucleus (FN) motor neurons innervating large facial whiskers in the rat. Using lentivirus-based GFP expression as a novel tool for axonal tracing, we demonstrate, both at the light and electron microscopy level, the existence of monosynaptic VMC-to-FN motor neuron connections. This corticomotoneuronal pathway is likely to contribute to VMC control of whisker movements, which, until now, were believed to be regulated solely via oligosynaptic VMC pathways, activity of the brainstem central pattern generator (CPG) neurons, and brainstem trigeminal sensorimotor loop (Hattox et al., 2002, 2003).

\section{Materials and Methods}

Production of recombinant lentiviruses. Production of lentiviruses was performed as described previously (Dittgen et al., 2004). Pyramidal neuron-specific expression of GFP was achieved from $1.3 \mathrm{~kb}$ mouse $\alpha$-calcium/calmodulin-dependent protein kinase II ( $\alpha$-CaMKII) promoter construct Flip/CaMKII promoter of $1.3 \mathrm{~kb} / \mathrm{GFP} /$ woodchuck posttranscriptional regulatory element [FCK(1.3)GW] (Dittgen et al., 2004). The synaptophysin-green fluorescent protein (GFP) coding sequence was subcloned under the control of the $\alpha$-CaMKII promoter, creating a construct FCK(1.3)GsynphyW.

VMC microstimulation, video-monitoring of whisker movements, and viral delivery. All animal experiments were performed according to the animal welfare guidelines of the Max Planck Society. We used standard surgical and electrophysiological techniques (Brecht et al., 2004a). Animals were anesthetized with ketamine hydrochloride $(90 \mathrm{mg} / \mathrm{kg}$, i.p. $) /$ xylazine ( $5 \mathrm{mg} / \mathrm{kg}$, i.p.) for surgical anesthesia. All pressure points and the skin incision were infused with lindocain. During M1 stimulation, animals received supplemental doses of ketamine $(20 \mathrm{mg} / \mathrm{kg})$ and acepromazine $(0.02 \mathrm{mg} / \mathrm{kg}$, i.m. $)$ as needed. Vibrissa motor cortex [anterior (A) 1.0 to A2.5, lateral (L) 1.0 to $\mathrm{L} 2.5 \mathrm{~mm}$ relative to bregma] was exposed, and extracellular intracortical microstimulation (ICMS) was applied. ICMS was only effective in evoking whisker movements when the animals were lightly anesthetized and displayed at least some minimal level of spontaneous whisker movements with amplitudes $>0.5^{\circ}$. The stimulation consisted of 100 0.3-ms-long monophasic cathodal pulses applied at a frequency of $333 \mathrm{~Hz}$, resulting in a 300 -ms-stimulation train. Current was delivered through a stimulus isolator connected to an extracellular stimulation pipette (tip resistance, $0.5-1 \mathrm{M} \Omega$ ) at a depth of 1500 $\mu \mathrm{m}$ below the pial surface at a rate of 0.1 or $0.2 \mathrm{~Hz}$. The usual coordinates for stimulation sites in this study were $1.5 \mathrm{~mm}$ anterior and $1.5 \mathrm{~mm}$ lateral from bregma. In all cases, stimulation at these coordinates with high-frequency stimulation trains $(333 \mathrm{~Hz}$ pulse repetition rate) led to backward whisker movements, indicating that we were targeting the large "retraction area" of VMC (Haiss and Schwarz, 2005). At four sites, we also tested stimulation frequencies of 10, 20, 50, and $100 \mathrm{~Hz}$. Consistent with previous observations (Brecht et al., 2004b), at three sites, we noted a reversal of movement direction at low frequencies (i.e., slow forward movements were evoked at $10 \mathrm{and} /$ or $20 \mathrm{~Hz}$ ). We videotaped whisker movements from the top, and whiskers of interest were labeled with reflex foil; the angular movements of whiskers of interest were quantified by a video-tracking system (Whiskerwatcher; Arrington Research, Scottsdale, AZ).

Single viral injections ( $<100 \mathrm{nl}$ per injection; total of nine animals) were made using the stereotaxic coordinates determined by the VMC microstimulation via a sharp glass capillary in a stereotaxic frame for small animals (David Kopf Instruments, Tujunga, CA) (Dittgen et al., 2004). Multiple injections ( $<100 \mathrm{nl}$ per injection; total of two animals) were made bilaterally using coordinates A1.2, A1.5, and A2.2 and L1.5 $\mathrm{mm}$ relative to bregma (depth, 1.5 and $1.2 \mathrm{~mm}$ for each site; six injections per hemisphere in total). In all cases, the GFP intrinsic signal was enhanced by staining with anti-GFP antibody (Molecular Probes, Eugene, OR). In three pilot experiments, the secondary horseradish peroxidase (HRP)-conjugated antibody was developed by the diaminobenzidine
(DAB) reaction (Vector Laboratories, Burlingame, CA). In all three animals, sparse labeled projections were detected in the lateral FN identified by Nissl staining (data not shown). To enhance the signal-to-noise ratio, we then switched to secondary FITC-conjugated antibody, which allowed us to analyze the labeling by confocal microscopy (see below). Four animals with GFP expression and two animals with synaptophysinGFP were processed this way. All light microscopy results presented here refer to the FITC-based experiments.

Electron microscopy. Rats were transcardially perfused under ketaminexylazine anesthesia with paraformaldehyde (PFA) fixative at variable $\mathrm{pH}$ levels ( $4 \%$ PFA in $2 \%$ sodium acetate, $\mathrm{pH}$ 6.5, followed by $4 \%$ PFA in 0.1 $\mathrm{M}$ sodium carbonate-sodium bicarbonate buffer with glutaraldehyde $0.02 \%, \mathrm{pH} 11$ ) (Berod et al., 1981). After perfusion, the brains were dissected and brainstem blocks containing the FN were postfixed for 2-3 $\mathrm{h}$ in fixative solution without glutaraldehyde at room temperature (RT), washed in PBS, and sectioned coronally at $50 \mu \mathrm{m}$. For electron microscopy (EM) immunohistochemistry, selected sections containing the FN were extensively rinsed in PBS and preincubated in 5\% normal goat serum (NGS; Sigma, St. Louis, MO) in PBS for $2 \mathrm{~h}$ at RT. Next, the sections were incubated with polyclonal rabbit anti-GFP primary antibody (rabbit anti-GFP, 1:10.000; Molecular Probes) in PBS with $0.04 \%$ Triton X-100 and $1 \%$ NGS for $72 \mathrm{~h}$ at $4^{\circ} \mathrm{C}$ with light agitation, followed by $6 \times 10$ min washes in PBS and incubation with biotinylated secondary antibody (goat anti-rabbit IgG 1:500; Vector Laboratories) overnight at $4^{\circ} \mathrm{C}$. After washing in PBS $(6 \times 10 \mathrm{~min})$, the sections were incubated in $\mathrm{ABC}$ complex (Vector Laboratories) for $2-3 \mathrm{~h}$ at RT. Immunolabeling was revealed by the glucose oxidase (GOD)-DAB method (Zaborszky and Heimer, 1989). Intensification of the immunoreaction product was performed using nickel-intensification (Liposits et al., 1986; Zaborszky and Heimer, 1989) of the GOD-DAB reaction product. Sections containing FN were treated with osmium tetroxide ( $1 \%$ in $0.1 \mathrm{M}$ PBS) for $1 \mathrm{~h}$ at $\mathrm{RT}$, washed for $15 \mathrm{~min}$ in bidistilled water, and dehydrated in a series of increasing concentrations of ethanol and then propylene oxide. After epon-propylene oxide (1:1) overnight infiltration, the sections were flat embedded in epon and cured at $60^{\circ} \mathrm{C}$ for $48 \mathrm{~h}$. Next, the lateral portions of the FN were cut out from the flat-embedded sections and reembedded onto epon blocks. First semithin $(0.5-1 \mu \mathrm{m})$ and then ultrathin sections $(\sim 60 \mathrm{~nm})$ were cut from the blocks. Serial ultrathin sections were cut with a Reichert-Jung ultramicrotome, collected on Pioloformcoated single-slot cooper grids and contrasted with uranyl acetate and lead citrate (Reynolds, 1963), and examined with a Zeiss EM 10C electron microscope (Zeiss, Thornwood, NY). In total, $>50$ ultrathin sections were analyzed. On average, one to four immunopositive axonal terminals were detected in synaptic contacts with proximal dendrite/cell body of FN neurons per single ultrathin section, and the majority of recognized synaptic appositions could be traced through consecutive serial sections. More detailed quantitative analysis of the immunopositive profiles was not performed, because our study was restricted to an ultrastructural confirmation of the presence of synaptic contacts between GFP-immunopositive terminals and their postsynaptic sites in the FN.

Back-labeling of facial motor neurons. Small skin incisions were made in front of the "best whisker" (the whisker that showed the largest movements evoked by cortical microstimulation) in ketamine-xylazineanesthetized animals to expose the whisker follicle. Care was taken to open the access enough to inject the dye to the bottom of the follicle, next to an exposed intrinsic muscle without causing injury. Using glass capillaries, 3-5 $\mu \mathrm{l}$ of $3 \%$ suspension of diamidino yellow (DY) or true blue (TB) (Sigma) was slowly injected bilaterally into follicles of the following whiskers: D1 (DY), D3 (DY), and B3 (TB) as described previously (Klein and Rhoades, 1985). Five days later, rats were killed by an overdose of halothane and were transcardially perfused with PBS followed by $4 \%$ paraformaldehyde in PBS, pH 7.4. Brains were removed and postfixed overnight at $4 \mathrm{C}^{\circ}$, washed in PBS ( $\left.1 \mathrm{~h} ; \mathrm{RT}\right)$, and embedded in $4 \%$ agarose.

Immunohistochemistry, confocal microscopy, and quantitation of the FN labeling. Serial frontal sections $(100 \mu \mathrm{m})$ of whole brains were cut on a vibratome (Leica, Nussloch, Germany) and collected in PBS. Floating sections were preincubated at RT in preblock solution (PBS, pH 7.4, 1\% Triton X-100, 5\% NGS), followed by overnight incubation with rabbit anti-GFP antibody (diluted 1:10,000; Molecular Probes) and mouse anti- 
neuron-specific nuclear protein $(\mathrm{NeuN})$ (diluted 1:1000; Chemicon, Temecula, CA) in PBS containing $0.5 \%$ Triton X-100 and 1\% NGS. The next day, sections were washed with PBS (three times for $10 \mathrm{~min}$ ) and incubated for $1 \mathrm{~h}$ at RT with FITC- and Texas Red-conjugated antirabbit and anti-mouse IgG antibodies (Jackson ImmunoResearch, West Grove, PA) diluted 1:200 in PBS containing 0.5\% Triton X-100 and $1 \%$ NGS. Sections were then washed in PBS (three times for $10 \mathrm{~min}$ ), affixed onto slides, and coverslipped with Mowiol (Sigma). All images were acquired on a confocal microscope Leica TCS NT microscope; digitized images were analyzed using Adobe Photoshop (Adobe Systems, Mountain View, CA). For quantitations, the FN area of each section was outlined on images of anti-NeuN staining, which visualized the large soma motor neuron morphology. Next, this outline was overlaid on corresponding images of anti-GFP labeling, and individual fibers that were detected within the FN outline were counted and measured. Adobe Photoshop (Adobe Systems) was used for FN selection and overlays; ImageJ was used for fiber length measurements. Because the count of the axonal fibers was done from a large number of sections (130 sections in total; from four animals with single injections and one with multiple injections), we performed the analysis from images collected on an epifluorescent rather than con-

focal microscope, with $10 \times$ objective and digital $2 \times$ magnification. By this approach, we were able to straightforwardly analyze data from each section in one image; however, because of the thickness of the sections $(100 \mu \mathrm{m})$, some fibers were too far out of focus to be reliably traced. This resulted in some underestimation of the actual values for the total length of the fibers, but it did not influence the ipsilateral and contralateral ratios given in the Results. The sums of the total fiber length from both hemispheres were $161,123,3787$, and $3103 \mu \mathrm{m}$ for each animal with a single injection site and $9322 \mu \mathrm{m}$ for one multiply injected animal (a second animal with the same multiple injections was used for the EM analysis).

\section{Results}

Targeted infection of deep-layer pyramidal neurons of the vibrissa motor cortex

Targeted GFP-based labeling of pyramidal neurons in layer 5 of the VMC was achieved in two steps. First, VMC was identified by electrical microstimulation and video-monitoring of the movements of the whiskers (Fig. 1A). Second, a small volume $(<100$ $\mathrm{nl}$ ) of lentivirus-expressing GFP under the control of the pyramidal neuron-specific $\alpha$-CaMKII promoter construct FCK(1.3)GW (Dittgen et al., 2004) was injected into the VMC using stereotaxic coordinates determined by microstimulation. As analyzed 4 weeks after the viral injection, the VMC region containing the somata of infected neurons was mainly in layer 5 of the agranular medial area $\left(\mathrm{AG}_{\mathrm{m}}\right)$ (Fig. $\left.1 \mathrm{~B}\right) ; \mathrm{AG}_{\mathrm{m}}$ is the cytoarchitectonic subdivision of M1 corresponding to the VMC (Brecht et al., 2004b). The infected area was limited to an approximately spherical region, with a diameter of $\sim 500 \mu \mathrm{m}$ (see Discussion for the determinants of the size of the infected region). The morphology of the labeled neurons was normal without any signs of cellular degeneration. This observation agrees with numerous previous studies documenting that long-term expression of GFP from lentiviral vectors does not alter the morphology or physiology of the infected neurons (Naldini et al., 1996; Lois et al., 2002; Dittgen et al., 2004).

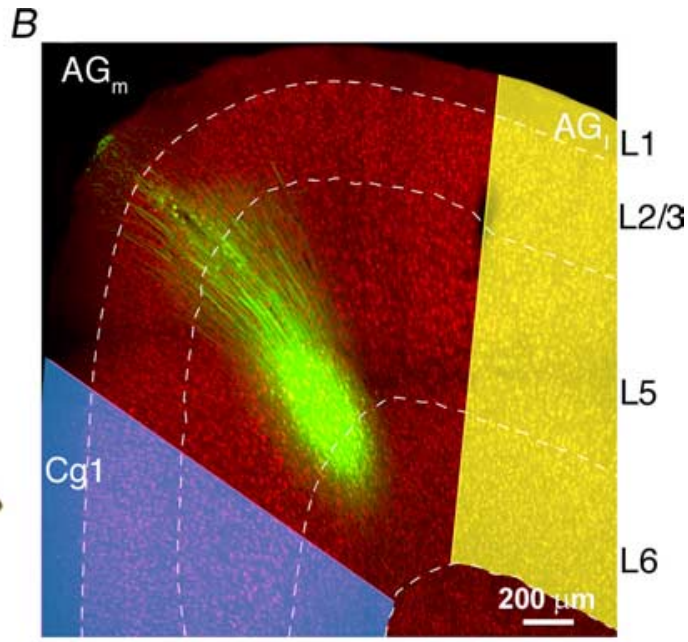

Figure 1. Lentivirus-based GFP labeling of VMC layer 5 pyramidal neurons. A, Top, Flat map of rat primary motor cortex (M1);

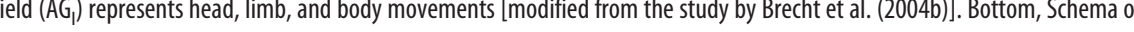
stack of four confocal sections, separated by $1 \mu \mathrm{m}$. The neighboring $\mathrm{Cg} 1$ and $\mathrm{AG}$, regions are shown overlaid with the same color coding as that used in the M1 map in $\boldsymbol{A}$.

VMC pyramidal neurons project to the lateral part of the facial nucleus, near whisker-innervating motor neurons For the initial analysis of putative VMC-to-FN projections, the location of the FN was determined by morphological identification of facial motor neurons stained with antibodies against the neuronal marker NeuN (Fig. 2 B) (note that somata of facial motor neurons are $\sim 30 \mu \mathrm{m}$ in diameter, whereas the cells in the surrounding perifacial nucleus region are of considerable smaller size, 10-15 $\mu \mathrm{m})$. Appreciably, GFP-positive fibers were located in close proximity to the large NeuN-positive motor neurons in the lateral portion of the FN (Fig. $2 B, C$ ), the FN subnucleus, which contains motor neurons innervating facial whisker muscles (Klein and Rhoades, 1985; Semba and Egger, 1986; Hattox et al., 2002). GFP labeling was detected in the lateral FN contralaterally as well as ipsilaterally to the injection site, with denser projections on the contralateral side. In the four animals with GFP-labeled VMC projections, including the three animals with retrograde labeling of motor neurons described below, the number as well as the total length of fibers in the contralateral FN was approximately threefold higher compared with the ipsilateral side: the contralateral-to-ipsilateral ratio of the total number of fibers detected was $3.5 \pm 1.6$, and that of the total fiber length was $3.1 \pm 1.2$ (mean \pm SD) (see Materials and Methods). In addition, in agreement with previous reports (Miyashita et al., 1994; Hattox et al., 2002), dense axonal projections were found in the brainstem reticular formation, a region that contains numerous premotor neurons projecting onto the facial motor neurons (Fig. $2 D-F)$.

Next, we examined the topographic specificity of the VMC projections within $\mathrm{FN}$, with respect to whisker-innervating motor neurons. First, injections of the GFP-expressing lentivirus were targeted to VMC sites mapped by intracortical microstimulations. Four weeks later, a retrograde axonal tracer, either TB or DY, was injected next to the follicle of the whisker that showed the largest movements during the microstimulation (termed the best 
$A$
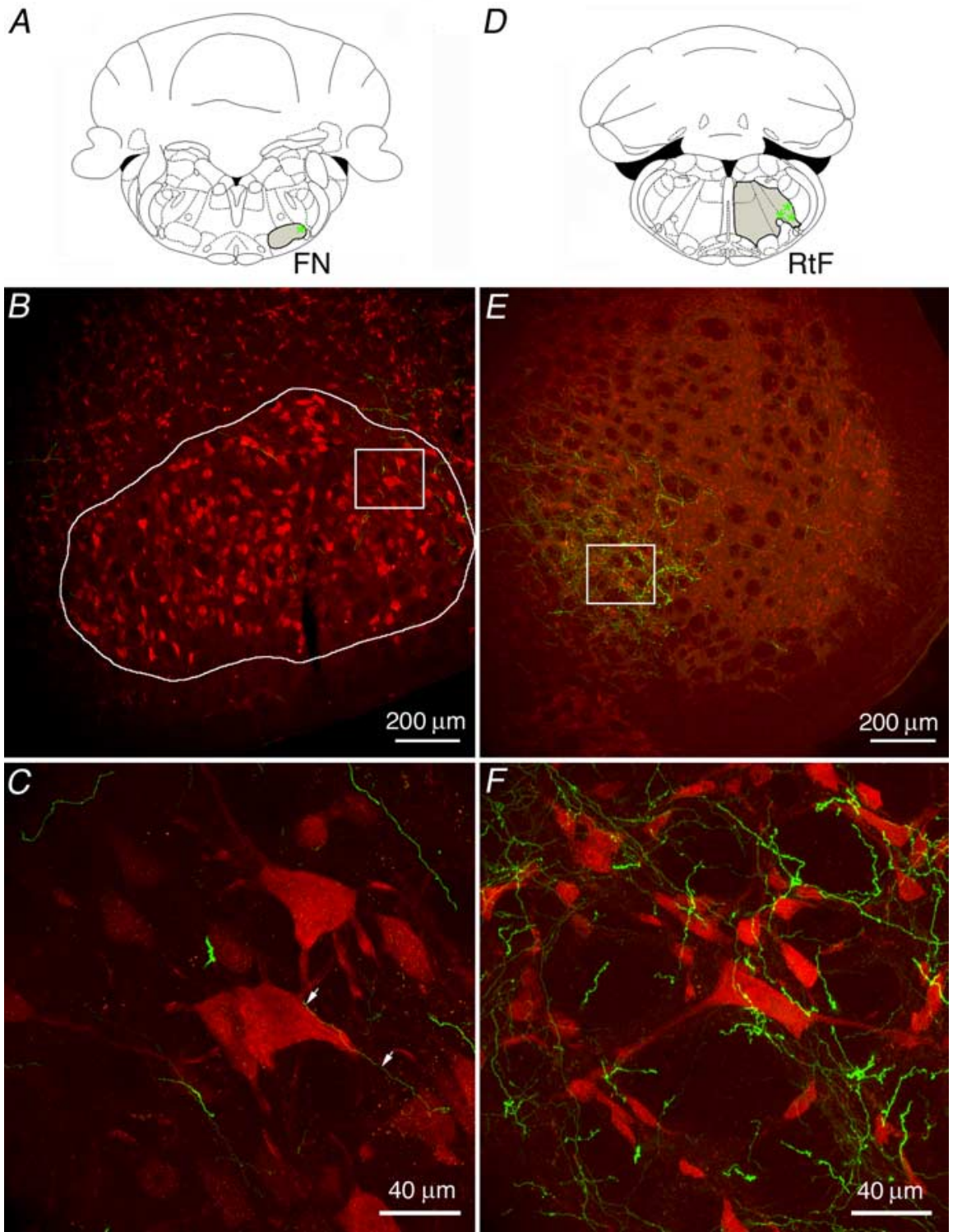

Figure 2. VMC axonal fibers in the lateral FN and reticular formation. $A$, Topography of GFP-positive fibers (green asterisk) in the lateral FN [schema adapted from the study by Paxinos and Watson (1998); coronal diagram 64; bregma, $-11.00 \mathrm{~mm}$ ]. $\boldsymbol{B}$, Distribution of GFP-positive fibers in the lateral FN. The FN area (outlined by a white line) was determined based on morphological identification of facial motor neurons stained with anti-NeuN antibodies. C, GFP-positive fibers in the FN at high magnification. The image is an enlargement from the area marked by a square outline in B. GFP-positive fibers were found in close apposition to large NeuN-positive facial motor neurons (arrows). D, Topography of GFP-positive fibers in the intermediate and parvocellular reticular formation (RtF; schema as in $A$; coronal diagram 67 ; bregma, $-11.80 \mathrm{~mm}$; three asterisks indicate dense labeling) (Paxinos and Watson, 1998). E, GFP-positive axons make a dense plexus surrounding NeuN-positive neurons in the parvocellular reticular nucleus. $\boldsymbol{F}$, High magnification of the fibers within the area marked by a square outline in $\boldsymbol{E}$. In $\boldsymbol{B}-\boldsymbol{E}$, brainstem sections were stained by immunohistochemistry with anti-GFP and anti-NeuN antibodies, followed by FITC and Texas Red-conjugated secondary antibodies. The images are maximal projection stacks of $102,31,68$, and 20 confocal sections separated by $0.48,1.0$, 1.0 , and $1.0 \mu \mathrm{m}$ in $\boldsymbol{B}, \boldsymbol{C}, \boldsymbol{E}$, and $\boldsymbol{F}$, respectively.

whisker), as well as to the same ipsilateral whisker. Three to $5 \mathrm{~d}$ later, the animals were killed and analyzed. As reported previously (Klein and Rhoades, 1985), TB-positive (Fig. 3A) or DYpositive (Fig. $3 D$ ) motor neurons were located in the lateral FN. GFP-positive axons of the VMC were found near the retrogradely labeled whisker motor neurons and often showed branching in the vicinity of the labeled somata (Fig. $3 B, E$ ). The lengths of facial motor neuron dendrites were estimated to be $250-500 \mu \mathrm{m}$ (Friauf, 1986), and thus the presence of GFP-labeled fibers within a
$250 \mu \mathrm{m}$ radius suggests that the motor neuron may be innervated by the VMC axons. In two of the three animals analyzed, almost all of the back-labeled motor neurons contained VMC fibers in the 250$\mu \mathrm{m}$-radius area, in both contralateral and ipsilateral FN (in total, 105 cells labeled; $90 \%$ of cells colocalized with fibers). In the third animal, the VMC projections in the FN were sparser, and, on average, $40 \%$ of the back-labeled facial motor neurons (33 cells labeled) had nearby GFP-labeled fibers.

\section{VMC axons form synapses on the lateral} FN motor neurons

GFP-labeled VMC axons showed numerous bouton-like varicosities within the lateral FN, suggesting that the axons form synapses on facial motor neurons (Fig. $3 C, F)$. Furthermore, lentivirus-based expression of GFP-tagged synaptophysin, a presynaptic protein abundantly present at the synaptic vesicles (Wiedenmann and Franke, 1985) from electrophysiologically mapped VMC, resulted in GFP-positive puncta-like labeling exclusively in the lateral part of the FN, in close proximity to NeuN-immunoreactive motor neurons (Fig. 4C-E). Because synaptophysin-GFP is well targeted to synaptic vesicles, effectively acting as a presynaptic terminal marker (Nakata et al., 1998; De Paola et al., 2003; Wimmer et al., 2004), the presence of such labeling in the lateral FN provides additional evidence for direct VMC innervation of facial motor neurons controlling whisker movements.

Finally, we performed EM of GFPlabeled VMC projections in the lateral FN to establish unequivocal anatomical evidence for a monosynaptic corticomotoneuronal pathway. To increase the level of FN labeling, GFP-expressing lentiviruses were injected bilaterally at three VMC sites (see Materials and Methods). As shown in Figure 5, a larger infection of VMC neurons resulted in denser projections in the lateral FN, with a threefold to ninefold increase in the total length of fibers detected in the FN compared with the single VMC injections (see Materials and Methods). For the EM analysis, after dissection of the lateral FN subnucleus, FN motor neurons were further identified by a round nucleus enriched with heterochromatin and containing one nucleolus, large accumulations of granular endoplasmatic reticulum, closely packed cisterns of the Golgi apparatus, and numerous mitochondria and multivesicular bodies (Senba and Tohyama, 1983; Mogoseanu et al., 1994) (Fig. 6A). Importantly, GFP-positive axon terminals containing spherical vesicles were readily found synapsing onto perikarya (Fig. $6 A, B$ ) and dendrites (Fig. $6 C, D$ ) of FN motor neurons. 


\section{Discussion}

Our study documents the existence of a monosynaptic VMC-to-FN pathway in a rat. This conclusion rests on the following observations: (1) the presence of GFPlabeled axons in the lateral FN after spatially restricted GFP expression at physiologically identified VMC sites; (2) the topographic specificity of the axonal projections, targeting lateral $\mathrm{FN}$-containing back-labeled motor neurons representing the best whisker of the VMC site; (3) the presence of VMC axonal varicosities (boutons) and GFP-synaptophysin labeling, both strongly suggesting presynaptic terminals, in the lateral FN; and (4) the demonstration of VMC GFP-labeled synaptic contacts onto motor neurons in the lateral portion of the FN by electron microscopy. We reason that confocal microscopy-based analysis of GFPlabeled axonal projections and GFPsynaptophysin-labeled putative axonal terminals can serve as a highly specific method for detection of presynaptic terminals, providing a less-demanding alternative to EM.

\section{The use of lentiviral vectors for axonal tracing}

Lentiviruses infect postmitotic cells, such as mammalian neurons, and stably integrate into the host cell genome (Kay et al., 2001; Kootstra and Verma, 2003). Because the extracellular space (ECS) in the vertebrate CNS has been estimated to be $<40$ nm (Van Harreveld, 1972; Hrabetova and Nicholson, 2002) and the human immunodeficiency virus-1 particles [both wildtype and vesicular stomatitis virus glycoprotein-G (VSV-G) pseudotyped] are $>100 \mathrm{~nm}$ in diameter (International Committee on Taxonomy of Viruses database; www.ncbi.nlm.nih.gov/ICTVdb) (Desmaris et al., 2001), the large size of the viral particles should strongly limit their diffusion in the brain ECS. This is likely the reason for the observed, uniformly re-

stricted size of the infected cortical region $(<500 \mu \mathrm{m}$ sphere $)$ after a single viral injection, even when using high-viral titers (Fig. 1). Furthermore, we did not observe any infected neurons in brain regions that project to the VMC, indicating that axonal uptake and/or retrograde transport is very rare for VSVG-coated lentiviruses [a similar observation was described by Blömer et al. (1997)]. Together, this shows that targeted injection of lentiviruses into a brain region of choice in living animals can be used for transgenic labeling of the infected neurons with high-spatial control.

Within the infected area, additional control over the cell specificity of the transgenic expression is given by the selection of an internal polymerase II promoter, which drives expression of the gene of interest in the lentiviral vector. We recently $\boldsymbol{E}$, respectively.

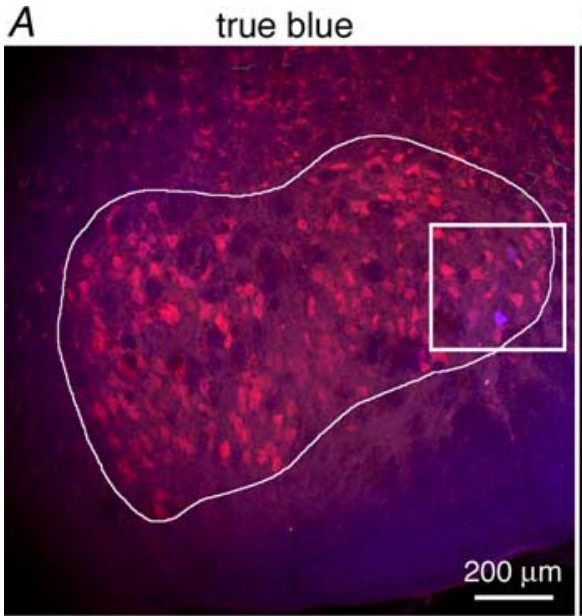

$D \quad$ diamidino yellow
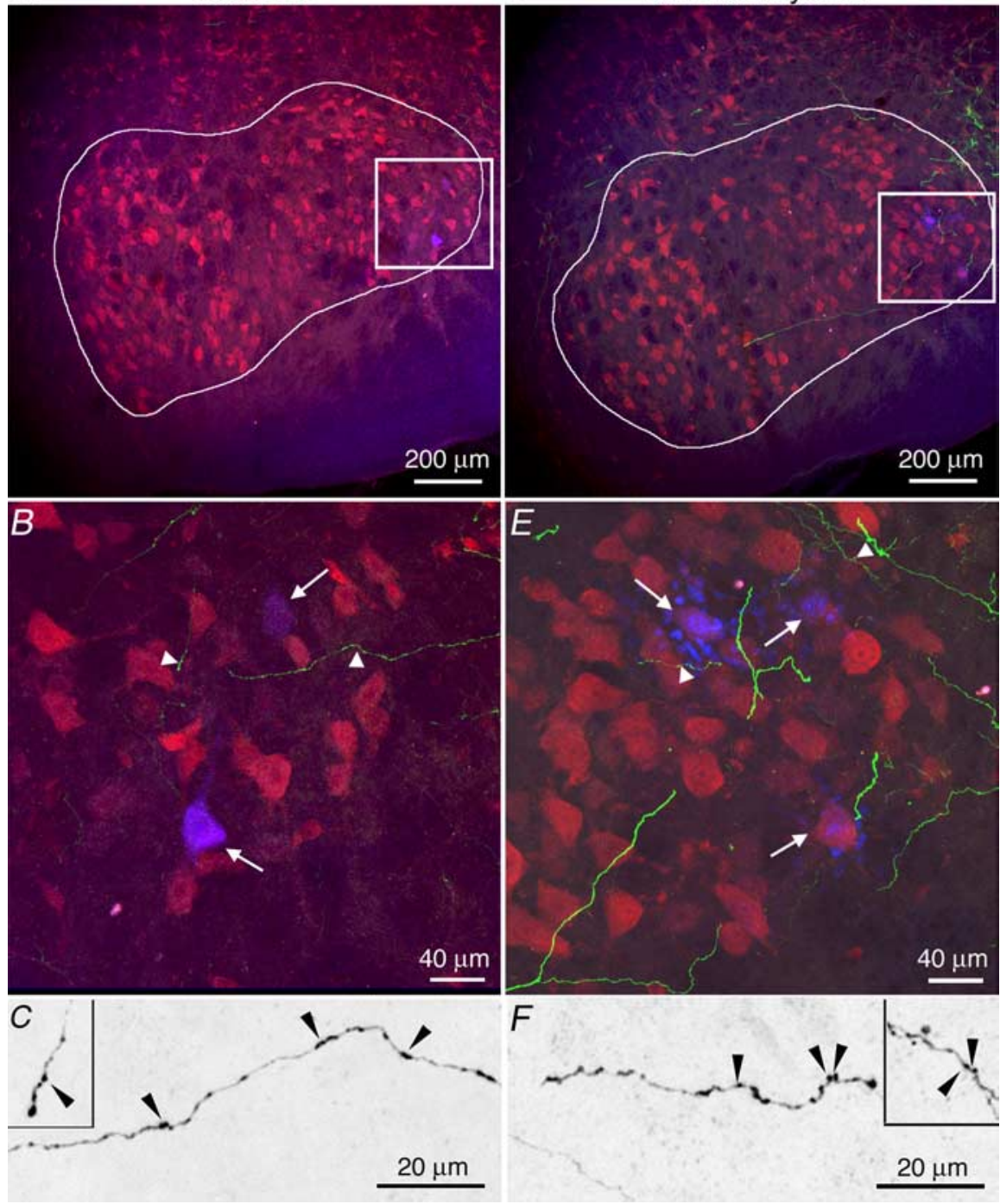

Figure 3. VMC axons target whisker-innervating motor neurons identified by axonal back-labeling. A, Overview of FN (outlined by a white line) with neuronal somata and GFP labeling as in Figure 2, as well as back-labeling of facial motor neurons with along GFP-positive fibers enlarged from $\boldsymbol{B}$ (images were changed to grayscale and inverted to enhance contrast). $\boldsymbol{D}-\boldsymbol{F}$ whisker D3. The images are maximal projection stacks of $46,37,81$, and 47 confocal sections separated by $1.0 \mu \mathrm{m}$ in $\boldsymbol{A}, \boldsymbol{B}, \boldsymbol{D}$, and

introduced a $1.3 \mathrm{~kb}$ fragment of the $\alpha$-CaMKII promoter into the self-inactivating vector to achieve efficient expression in cortical pyramidal neurons (Dittgen et al., 2004). Here, the use of this vector, in combination with intracortical microstimulation to identify the VMC area, allowed us to transgenically label anatomically and functionally defined population of cortical pyramidal neurons and to trace their axonal projections at a distance of $>1.5 \mathrm{~cm}$ from the injection site after 4 weeks of GFP expression [note that closer projections may be analyzed after shorter time intervals, because the onset of GFP expression is quite rapid with strong labeling of dendritic arbors and axon collaterals already at 1 week after infection (Dittgen et al., 2004)]. Clearly, lentivirus-based axonal tracing can be applied to other brain regions and/or other cell types by stereotaxic 

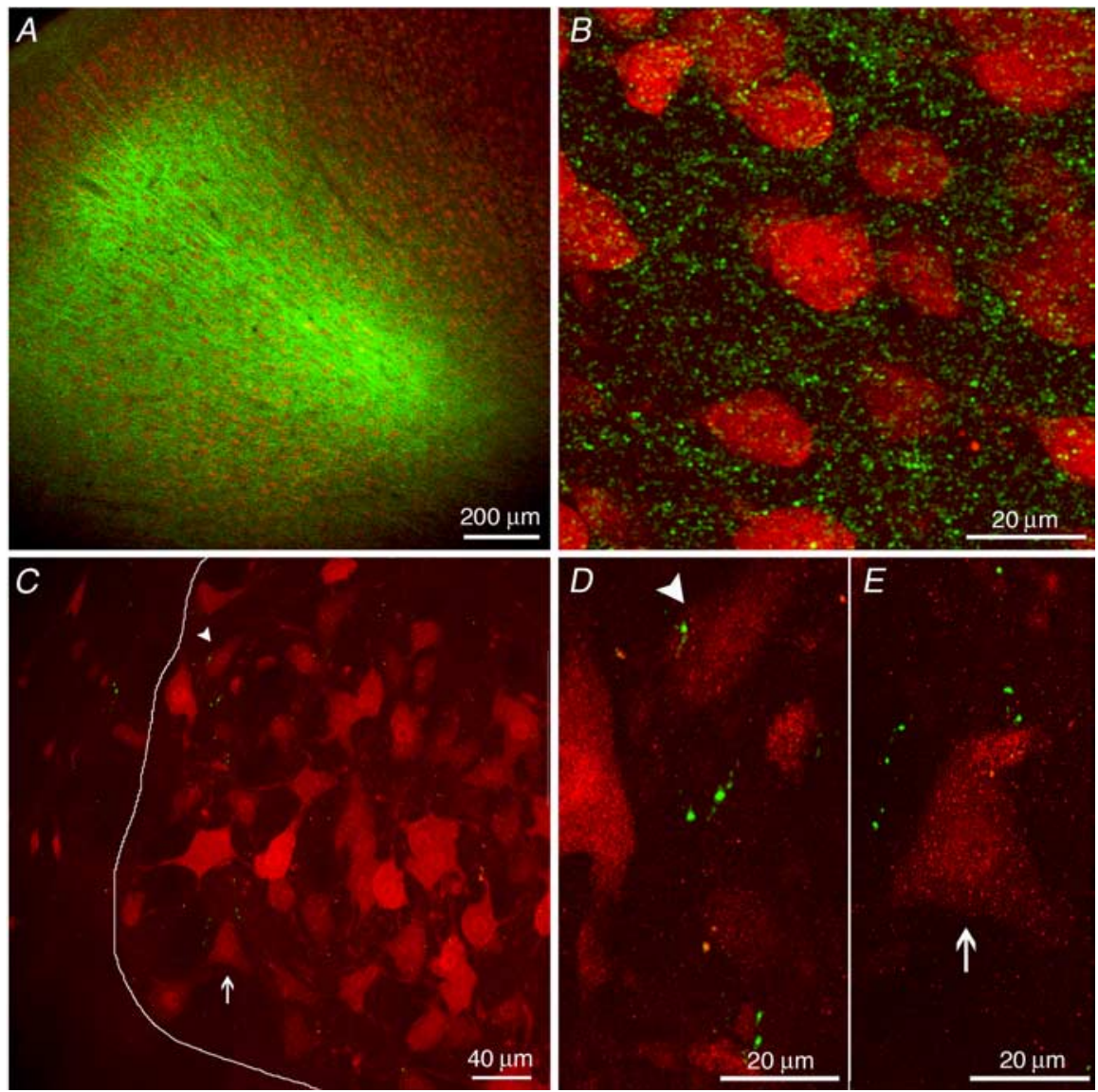

Figure 4. Synaptophysin-GFP labeling of presynaptic terminals in the VMC and FN. A, Synaptophysin-GFP labeling at VMC injection site 4 weeks after lentivirus injection. Note the diffuse labeling pattern representing axonal terminals compared with GFP-only labeling of the injection site shown in Figure 1. B. High-magnification view of GFP-positive bouton-like puncta in the VMC taken from layer 2 area. $C$, The lateral portion of the FN (outlined by a white line) with synaptophysin-GFP labeling in close proximity to facial motor neurons marked by arrows and arrowheads. D, E, Cells from Cat high magnification, with corresponding arrows and arrowheads. Sections were stained as in Figure 2 . The images are maximal projection stacks of $9,17,18,18$, and 18 confocal sections separated by $1.0 \mu \mathrm{m}$ in $\boldsymbol{A}-\boldsymbol{E}$, respectively.
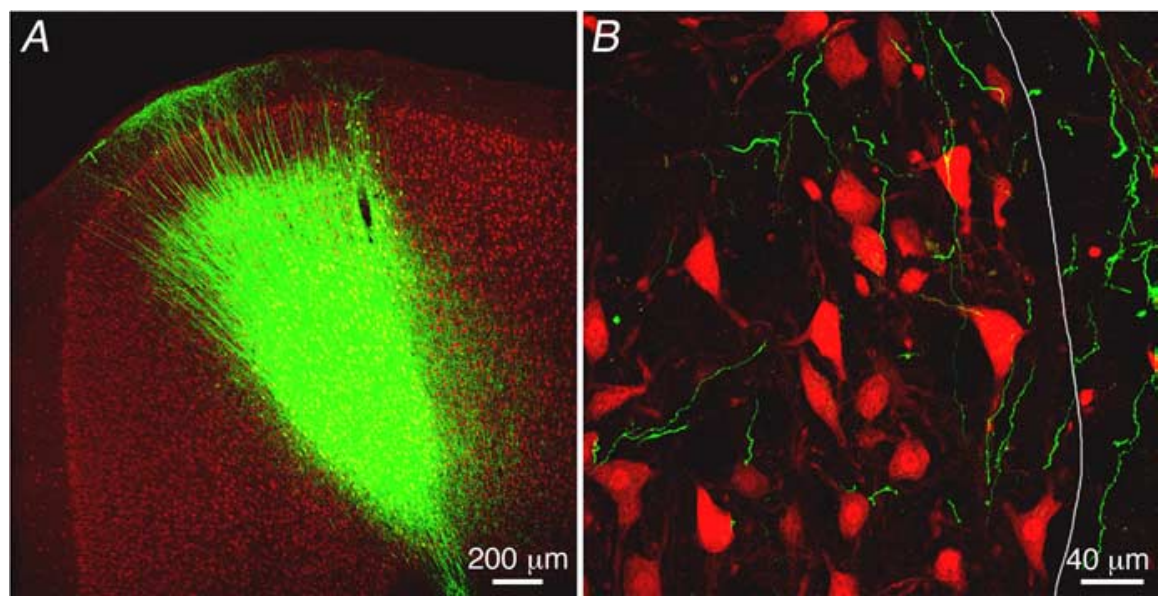

Figure 5. Dense infection of VMC results in a higher labeling of its projections in the FN. $A$, Confocal image of the injection area (4 weeks after lentivirus injection) showing dense GFP labeling in the $A G_{m}$ area (compare with Fig. $1 B$ showing only a single injection). The GFP signal was enhanced with anti-GFP followed by FITC-conjugated secondary antibodies; neuronal somata were stained with anti-NeuN followed by Texas Red-conjugated secondary antibodies. The image is a maximal projection stack of four confocal sections separated by $1 \mu \mathrm{m}$. $\boldsymbol{B}$, Lateral portion of the FN (outlined by white line) with dense GFP labeling in close proximity to facial motor neurons. The image is a maximal projection stack of 18 confocal sections separated by $1.0 \mu \mathrm{m}$. targeting and selection of an appropriate cell-specific recombinant promoter.

\section{Previous studies of VMC projections}

Two previous studies used anterograde tracing with Phaseolus vulgaris leucoagglutinin, wheat germ agglutinin, and biotinylated dextran amine to label and identify axonal projections from the rat VMC area (Miyashita et al., 1994; Hattox et al., 2002). Positive labeling in numerous premotor structures in the brainstem, metencephalon, and midbrain, together with the lack of labeling in the FN, led to a proposal that VMC regulates whisker movements only indirectly via oligosynaptic pathways (Miyashita et al., 1994; Hattox et al., 2002). We focused our analysis of VMC projections primarily on the brainstem. In agreement with the two previous studies, dense labeling was observed in the previously described areas, including the parvocellular, gigantocellular, and intermediate reticular formation. In our view, there are two main reasons why we were able to detect the VMC-to-FN pathway: (1) the lentivirusdriven long-term (4 week) expression was sufficient for diffusion of GFP or active transport of synaptophysin-GFP into distal axonal projections, and such genetic tracing may be more efficient in the labeling of fine distal braches compared with the classical anterograde tracers (see above); and (2) the antibody-based enhancement of the GFP signal coupled to FITC-based visualization allowed us to analyze the labeling by confocal microscopy, which provides higher sensitivity with better signal-to-noise ratio over light microscopy used with the traditional tracing methods. Furthermore, the combination of anti-GFP antibody followed by secondary HRP-conjugated antibody allowed us to verify the projections at the EM level.

\section{Corticomotoneuronal connectivity in primates and rodents}

Three corticomotoneuronal projections have been described in humans and in some nonhuman primates, each with a distinct motor function. Two direct pathways, one onto facial motor neurons innervating lower facial (especially perioral) muscles and the other onto hypoglossal motor neurons innervating tongue muscles, indicate a phylogenetic trend in the development of motor control over tongue and facial movements with respect to facial mimicry and (in humans) speech (Kuypers, 1958a,b; Jenny and Saper, 1987; Morecraft et al., 2001, 2004; Jürgens and Alipour, 2002). The third pathway, pro- 
jecting onto spinal motor neurons innervating arm and hand muscles, is believed to enable relatively independent (fractionated) movements of fingers (Kuypers, 1982). For example, in two species of New World monkeys with similar hands with pseudo-opposable thumbs, cebus but not squirrel monkeys are capable of performing independent finger movements (Costello and Fragaszy, 1988), and cebus but not squirrel monkeys have abundant direct corticospinal projections onto hand-innervating ventral horn motor neurons (Bortoff and Strick, 1993).

Although the VMC-to-FN pathway in rodents was not described previously, there are reports of corticomotoneuronal innervation in the rat corticospinal system (Elger et al., 1977; Liang et al., 1991). However, this evidence has been disputed previously (Lemon et al., 1991; Yang and Lemon, 2003; Alstermark et al., 2004). The corticomotoneuronal pathway described here relates specifically to facial motor neurons innervating muscles of large facial whiskers in the rat. We reason that the projections are quite abundant, because of the following: (1) single-viral injections, which labeled only a fraction of VMC deep layer neurons, resulted in positive lateral FN labeling in all animals injected (seven animals with GFP and two animals with synaptophysin-GFP expression); and (2) multiple VMC injections allowed detection of numerous synaptic contacts, including three synapses onto one dendrite detected in a single cross-section (Fig. 6C), by electron microcopy. The description of the VMC-to-FN pathway thus adds to our understanding of the complex wiring diagram of neural control in the vibrissal system (Kleinfeld et al., 1999; Hattox et al., 2002, 2003). We speculate that the evolution of the VMC-to-FN projections is related to the rich repertoire of whisker movements in rodents, whereas simple synchronous movements of whiskers are observed during free whisking, rats do complex movements of subsets of whiskers when they are in object contact (Welker, 1964; Wineski, 1985; Carvell and Simons, 1990; Sachdev et al., 2002, 2003). Similar relatively independent whisker movements can also be evoked by intracortical microstimulation (Izraeli and Porter, 1995; Brecht et al., 2004a,b). Thus, one can speculate that the direct VMC-to-FN pathway plays a role in the generation of complex whisker movements during tactile exploration. Clearly, the elaborate control of whisker movements, comprising monosynaptic (our study) and oligosynaptic (Miyashita et al., 1994; Hattox et al., 2002) corticomotoneuronal projections, brainstem CPG inputs (Hattox et al., 2003), and brainstem trigeminal sensorimotor loop (Nguyen and Kleinfeld, 2005), is likely to contribute to the remarkable tactile acuity of rat active touch in whisker-based texture, shape, and/or distance discriminations (Guic-Robles et al., 1989; Carvell and
Simons, 1990; Harvey et al., 2001; Krupa et al., 2001; Brecht et al., 2004b).

\section{References}

Alstermark B, Ogawa J, Isa T (2004) Lack of monosynaptic corticomotoneuronal EPSPs in rats: disynaptic EPSPs mediated via reticulospinal neurons and polysynaptic EPSPs via segmental interneurons. J Neurophysiol 91:1832-1839.

Berg RW, Kleinfeld D (2003) Rhythmic whisking by rat: retraction as well as protraction of the vibrissae is under active muscular control. J Neurophysiol 89:104-117.

Berod A, Hartman BK, Pujol JF (1981) Importance of fixation in immunohistochemistry: use of formaldehyde solutions at variable $\mathrm{pH}$ for the localization of tyrosine hydroxylase. J Histochem Cytochem 29:844-850.

Blömer U, Naldini L, Kafri T, Trono D, Verma IM, Gage FH (1997) Highly efficient and sustained gene transfer in adult neurons with a lentivirus vector. J Virol 71:6641-6649.

Bortoff GA, Strick PL (1993) Corticospinal terminations in two new-world 
primates: further evidence that corticomotoneuronal connections provide part of the neural substrate for manual dexterity. J Neurosci 13:5105-5118.

Brecht M, Preilowski B, Merzenich MM (1997) Functional architecture of the mystacial vibrissae. Behav Brain Res 84:81-97.

Brecht M, Schneider M, Sakmann B, Margrie TW (2004a) Whisker movements evoked by stimulation of single pyramidal cells in rat motor cortex. Nature 427:704-710.

Brecht M, Krauss A, Muhammad S, Sinai-Esfahani L, Bellanca S, Margrie TW (2004b) Organization of rat vibrissa motor cortex and adjacent areas according to cytoarchitectonics, microstimulation, and intracellular stimulation of identified cells. J Comp Neurol 479:360-373.

Carvell GE, Simons DJ (1990) Biometric analyses of vibrissal tactile discrimination in the rat. J Neurosci 10:2638-2648.

Costello MB, Fragaszy DM (1988) Prehension in Cebus and Saimiri: I. Grip type and hand preference. Am J Primatol 15:235-245.

De Paola V, Arber S, Caroni P (2003) AMPA receptors regulate dynamic equilibrium of presynaptic terminals in mature hippocampal networks. Nat Neurosci 6:491-500.

Desmaris N, Bosch A, Salaun C, Petit C, Prevost MC, Tordo N, Perrin P, Schwartz O, de Rocquigny H, Heard JM (2001) Production and neurotropism of lentivirus vectors pseudotyped with lyssavirus envelope glycoproteins. Mol Ther 4:149-156.

Dittgen T, Nimmerjahn A, Komai S, Licznerski P, Waters J, Margrie TW, Helmchen F, Denk W, Brecht M, Osten P (2004) Lentivirus-based genetic manipulations of cortical neurons and their optical and electrophysiological monitoring in vivo. Proc Natl Acad Sci USA 101:18206-18211.

Dörfl J (1982) The musculature of the mystacial vibrissae of the white mouse. J Anat 135:147-154.

Elger CE, Speckmann EJ, Caspers H, Janzen RW (1977) Cortico-spinal connections in the rat. I. Monosynaptic and polysynaptic responses of cervical motoneurons to epicortical stimulation. Exp Brain Res 28:385-404.

Friauf E (1986) Morphology of motoneurons in different subdivisions of the rat facial nucleus stained intracellularly with horseradish peroxidase. J Comp Neurol 253:231-241.

Guic-Robles E, Valdivieso C, Guajardo G (1989) Rats can learn a roughness discrimination using only their vibrissal system. Behav Brain Res 31:285-289.

Haiss F, Schwarz C (2005) Spatial segregation of different modes of movement control in the whisker representation of rat primary motor cortex. J Neurosci 25:1579-1587.

Hall RD, Lindholm EP (1974) Organization of motor and somatosensory neocortex in the albino rat. Brain Res 66:23-28.

Harvey MA, Bermejo R, Zeigler HP (2001) Discriminative whisking in the head-fixed rat: optoelectronic monitoring during tactile detection and discrimination tasks. Somatosens Mot Res 18:211-222.

Hattox A, Li Y, Keller A (2003) Serotonin regulates rhythmic whisking. Neuron 39:343-352.

Hattox AM, Priest CA, Keller A (2002) Functional circuitry involved in the regulation of whisker movements. J Comp Neurol 442:266-276.

Hrabetova S, Nicholson C (2002) Do electron micrographs underestimate gaps between brain cells? Soc Neurosci Abstr 28:505.517.

Izraeli R, Porter LL (1995) Vibrissal motor cortex in the rat: connections with the barrel field. Exp Brain Res 104:41-54.

Jenny AB, Saper CB (1987) Organization of the facial nucleus and corticofacial projection in the monkey: a reconsideration of the upper motor neuron facial palsy. Neurology 37:930-939.

Jin TE, Witzemann V, Brecht M (2004) Fiber types of the intrinsic whisker muscle and whisking behavior. J Neurosci 24:3386-3393.

Jürgens U, Alipour M (2002) A comparative study on the corticohypoglossal connections in primates, using biotin dextranamine. Neurosci Lett 328:245-248.

Kay MA, Glorioso JC, Naldini L (2001) Viral vectors for gene therapy: the art of turning infectious agents into vehicles of therapeutics. Nat Med $7: 33-40$.

Klein BG, Rhoades RW (1985) Representation of whisker follicle intrinsic musculature in the facial motor nucleus of the rat. J Comp Neurol 232:55-69.

Kleinfeld D, Berg RW, O’Connor SM (1999) Anatomical loops and their electrical dynamics in relation to whisking by rat. Somatosens Mot Res 16:69-88.

Kootstra NA, Verma IM (2003) Gene therapy with viral vectors. Annu Rev Pharmacol Toxicol 43:413-439.

Krupa DJ, Matell MS, Brisben AJ, Oliveira LM, Nicolelis MA (2001) Behavioral properties of the trigeminal somatosensory system in rats performing whisker-dependent tactile discriminations. J Neurosci 21:5752-5763.

Kuypers HG (1958a) Corticobular connexions to the pons and lower brainstem in man: an anatomical study. Brain 81:364-388.

Kuypers HG (1958b) Some projections from the peri-central cortex to the pons and lower brain stem in monkey and chimpanzee. J Comp Neurol 110:221-255.

Kuypers HG (1982) A new look at the organization of the motor system. Prog Brain Res 57:381-403.

Lee KJ, Woolsey TA (1975) A proportional relationship between peripheral innervation density and cortical neuron number in the somatosensory system of the mouse. Brain Res 99:349-353.

Lemon RN, Bennett KMB, Werner W (1991) The cortico-motor substrate for skilled movements of the primate hand. In: Tutorials on motor neuroscience (Stelmach GE, Requin J, eds), pp 477-495. Holland: Kluwer Academic.

Liang FY, Moret V, Wiesendanger M, Rouiller EM (1991) Corticomotoneuronal connections in the rat: evidence from double-labeling of motoneurons and corticospinal axon arborizations. J Comp Neurol 311:356-366.

Liposits Z, Sherman D, Phelix C, Paull WK (1986) A combined light and electron microscopic immunocytochemical method for the simultaneous localization of multiple tissue antigens. Tyrosine hydroxylase immunoreactive innervation of corticotropin releasing factor synthesizing neurons in the paraventricular nucleus of the rat. Histochemistry 85:95-106.

Lois C, Hong EJ, Pease S, Brown EJ, Baltimore D (2002) Germline transmission and tissue-specific expression of transgenes delivered by lentiviral vectors. Science 295:868-872.

Miyashita E, Keller A, Asanuma H (1994) Input-output organization of the rat vibrissal motor cortex. Exp Brain Res 99:223-232.

Mogoseanu D, Smith AD, Bolam JP (1994) Monosynaptic innervation of facial motoneurones by neurones of the parvicellular reticular formation. Exp Brain Res 101:427-438.

Morecraft RJ, Louie JL, Herrick JL, Stilwell-Morecraft KS (2001) Cortical innervation of the facial nucleus in the non-human primate: a new interpretation of the effects of stroke and related subtotal brain trauma on the muscles of facial expression. Brain 124:176-208.

Morecraft RJ, Stilwell-Morecraft KS, Rossing WR (2004) The motor cortex and facial expression: new insights from neuroscience. Neurologist 10:235-249.

Nakata T, Terada S, Hirokawa N (1998) Visualization of the dynamics of synaptic vesicle and plasma membrane proteins in living axons. J Cell Biol 140:659-674.

Naldini L, Blömer U, Gallay P, Ory D, Mulligan R, Gage FH, Verma IM, Trono D (1996) In vivo gene delivery and stable transduction of nondividing cells by a lentiviral vector. Science 272:263-267.

Nguyen QT, Kleinfeld D (2005) Positive feedback in a brainstem tactile sensorimotor loop. Neuron 45:447-457.

Paxinos G, Watson C (1998) The rat brain in stereotaxic coordinates. San Diego: Academic.

Porter R, Lemon R (1995) Corticospinal function and voluntary movement. In: Monographs of the physiological society. Gloucestershire, UK: Clarendon.

Reynolds ES (1963) The use of lead citrate at high pH as an electron-opaque stain in electron microscopy. J Cell Biol 17:208-212.

Sachdev RN, Sato T, Ebner FF (2002) Divergent movement of adjacent whiskers. J Neurophysiol 87:1440-1448.

Sachdev RN, Berg RW, Champney G, Kleinfeld D, Ebner FF (2003) Unilateral vibrissa contact: changes in amplitude but not timing of rhythmic whisking. Somatosens Mot Res 20:163-169.

Semba K, Egger MD (1986) The facial "motor" nerve of the rat: control of vibrissal movement and examination of motor and sensory components. J Comp Neurol 247:144-158.

Senba E, Tohyama M (1983) Leucine-enkephalin-containing neuron sys- 
tem in the facial nucleus of the rat with special reference to its fine structure. Brain Res 274:17-23.

Van Harreveld A (1972) The extracellular space in the vertebrate central nervous system. In: The structure and fuction of nervous tissue (Bourne GH, ed), pp 447-511. New York: Academic.

Welker WI (1964) Analysis of sniffing of the albino rat. Behaviour 22:223-244.

Wiedenmann B, Franke WW (1985) Identification and localization of synaptophysin, an integral membrane glycoprotein of $\mathrm{Mr}$ 38,000 characteristic of presynaptic vesicles. Cell 41:1017-1028.

Wimmer VC, Nevian T, Kuner T (2004) Targeted in vivo expression of proteins in the calyx of Held. Pflügers Arch 449:319-333.
Wineski LE (1985) Facial morphology and vibrissal movement in the golden hamster. J Morphol 183:199-217.

Wree A, Lutz B, Thole U (1992) Volumes of the cytoarchitectonic areas in the rat cerebral cortex. J Hirnforsch 33:545-548.

Yang HW, Lemon RN (2003) An electron microscopic examination of the corticospinal projection to the cervical spinal cord in the rat: lack of evidence for cortico-motoneuronal synapses. Exp Brain Res 149:458-469.

Zaborszky L, Heimer L (1989) Combinations of tracer techniques, especially HRP and PHA-L, with transmitter identification for correlated light and electron microscopic studies. In: Neuroanatomical tract tracing methods 2 plenum (Heimer L, Zaborszky L, eds), pp 49-96. New York: Plenum. 